\title{
Sleeping body temperatures in 3-4 month old infants
}

\author{
M P WAILOO, S A PETERSEN, ${ }^{*}$ H WHITTAKER, AND P GOODENOUGH \\ Departments of Child Health and *Physiology, University of Leicester
}

SUMMARY Rectal, skin, and ambient temperatures were continuously recorded overnight from 3-4 month old normal infants in their home cots under conditions of room temperature and wrapping chosen freely by parents. It was found that rectal temperature was above $37^{\circ} \mathrm{C}$ when infants were put down, but fell rapidly to $36.4^{\circ} \mathrm{C}$ within one and a half hours, then stabilised for a few hours before rising steadily. This pattern was tied more closely to the time of putting down than time of day. The extent and rate of temperature fall did not correlate with any feature of the thermal environment. We also found that skin temperature changed much less than rectal temperature over the night, and for the first two hours in the cot there was no relation between skin and rectal temperature. There is therefore a well organised, endogenous rhythm of temperature in 4 month old infants.

At some time during the first year of life, infants develop rhythmical changes in certain body functions based on a 24 hour cycle, ${ }^{1}$ whereby activities are at a peak during daytime wakefulness and decline during nightime with sleep. ${ }^{2}$ Body temperature, in line with other functions, tends to fall during the night and rise during the day to levels predetermined endogenously. ${ }^{3}{ }^{4}$ It is possible, however, that other factors in a home environment-for example, ambient temperature, quality of clothing, or infection-might influence body temperature to produce variation that might modify the normal circadian rhythm. Recently the findings of raised core temperature at necropsy in children who died suddenly ${ }^{5}$ have led to suggestions of fatal hyperpyrexia during sleep, ${ }^{6}$ although it is unlikely that postmortem findings accurately reflect the temperature state of live babies. We studied the pattern of core temperature during sleep in babies at the age of peak incidence of sudden deaths with relation to the home conditions that may significantly alter that pattern.

\section{Subjects and methods}

Information on a random sample of 98 normal, full term, singleton babies was collected at birth, and permission obtained from parents for the body temperature to be monitored for one night during sleep between the ages of 3-4 months. At this time each child was visited at home, weighed, skin fold thickness measured at the mid forearm region, and information obtained on feeding pattern and the recent health of the baby. Three thermistor probes were securely attached to the baby: (a) at the centre of the forehead; (b) on the abdomen $3 \mathrm{~cm}$ to the left of the umbilicus; and (c) into the rectum $5 \mathrm{~cm}$ from the anal margin. A fourth probe was fixed at the cot side to measure room temperature. All probes were connected to a battery powered Grant Squirrel Data Logger that was set to make recordings to the nearest $0 \cdot 1^{\circ} \mathrm{C}$ at one minute intervals throughout the night from 2200 to 0700 . Each probe was secured with sticking plaster to ensure good contact during body movement or bowel actions. Before use the probes and data loggers were tested for accuracy, firstly by placing them in water at known temperatures, and secondly by observing them on babies of similar age admitted to the ward.

The infants in the study were closely monitored during the night, both to check the security of the probes and to standardise readings against clinical thermometers at half hourly intervals. Parents were advised not to alter clothing, wrapping, and room heating practice, so that recording under normal conditions could be obtained.

Note was made of the number of quality of each item of clothing and wrapping, so that the thermal conductivity (tog value) could be calculated. ${ }^{7}$

A diary of the night's events was kept by parents recording the time the infant was put to bed, waking, crying, etc, and recordings commenced an hour or so before bedtime. All measurements were taken during December-March so that the widest 
variation in environmental and room temperatures would be obtained.

\section{SUBJECTS}

Of the 98 subjects recruited into the study 74 were eventually available for recording of body temperatures. Of the recordings made only those of at least eight hours duration showing no evidence of disturbance of the probes were selected for analysis; this gave 67 subjects with a full data set. The seven remaining subjects yielded only partial temperature records.

The subjects ranged in age from 9.5 to 19.5 weeks (standardised to a 40 week gestation) at the time of recording with a mean (SEM) age of $14.81(0.25)$ weeks. Only seven infants were outside the range of 12 to 18 weeks. The mean (SEM) body weight at monitoring was $6265(80) \mathrm{g}$ (range $4520-8100 \mathrm{~g}$ ) and the birth weight 3257 (50)g (range 2360-4340 g). They slept in cots in rooms whose minimum temperature ranged from $5.8^{\circ} \mathrm{C}$ to $22.4^{\circ} \mathrm{C}$ with a mean (SEM) of $13.95(0.44)^{\circ} \mathrm{C}$, and were clothed and wrapped to tog values ranging from $5 \cdot 1$ to $29 \cdot 2$ with a mean (SEM) of $14.7(0 \cdot 89)$. The factors influencing wrapping and parental choice of room temperature are the subject of a separate report. ${ }^{8}$

\section{Results}

\section{RECTAL TEMPERATURE}

The data logger recorded temperature to the nearest $0 \cdot 1^{\circ} \mathrm{C}$ at one minute intervals. Data were reduced to a 15 minute average and plotted over the period of recording. All but three of the plots showed the same characteristics. The three oddities showed a rise of rectal temperature, occasionally up to $38.5^{\circ} \mathrm{C}$, and were not included in the study, though as their number was so small their inclusion would not affect any conclusions reported here.

In the remaining 64 recordings rectal temperature was generally above $37^{\circ} \mathrm{C}$ before the infant was put down, and then fell rapidly within an hour of placing in the cot to stabilise at a value around $0.8^{\circ} \mathrm{C}$ lower. There were occasional further falls of $0 \cdot 1-0 \cdot 2^{\circ} \mathrm{C}$. After around five hours the rectal temperature rose steadily at a slower rate than its fall. Fig 1 shows examples of individual temperature records. Although the pattern is similar the timing varies by up to three hours either way, depending on the time of putting down.

Combining data across individuals at fixed times of night would therefore tend to obscure the pattern of change, because the patterns are not precisely time locked. Accordingly the records were all time shifted to superimpose the one event that did appear phase locked to the pattern, namely putting down in

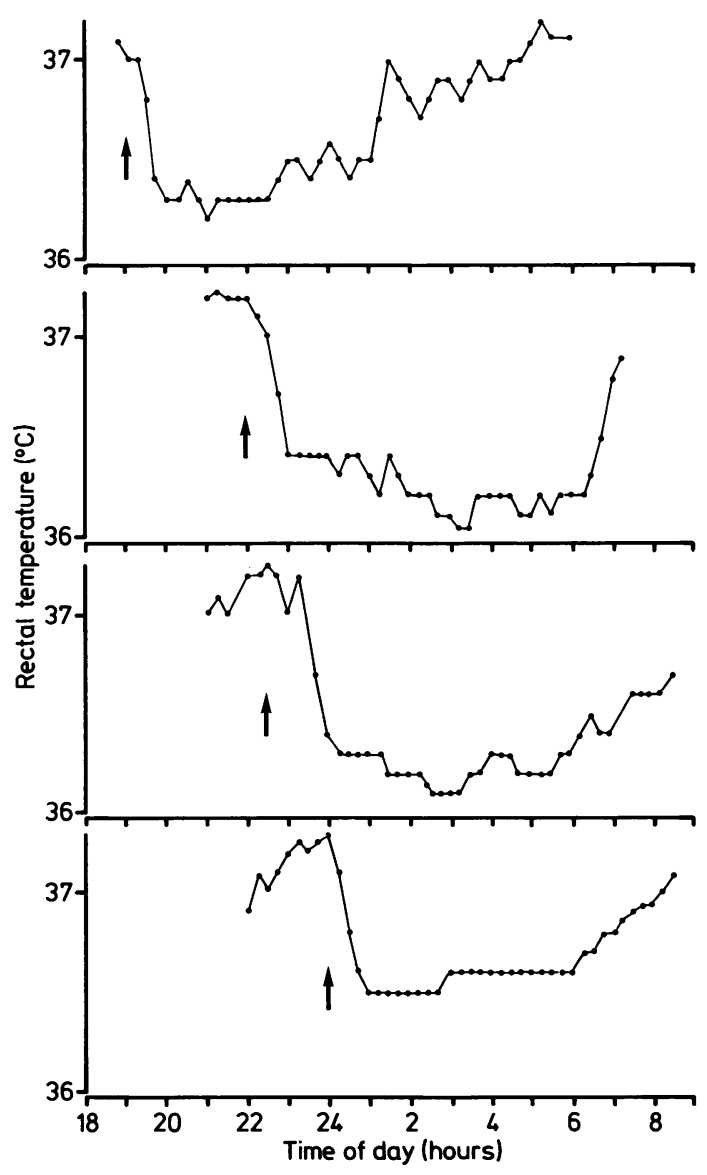

Fig 1 Examples of four continuous rectal temperature records from babies in their cots at night. Data are reduced to 15 minute averages from one minute samples. The arrows show bedtime.

the cot, producing a curve whose time axis is expressed relative to bedtime.

Fig 2 shows the normalised data. On the assumption of a normal distribution of temperature, $90 \%$ of infants would be expected to fall in the range shown by the shading. Data do not depart significantly from a normal distribution. The distribution is not, however, wholly symmetrical, particularly during the period of stable rectal temperature two to five hours after putting the baby down (fig 3 ). Temperatures below $35.9^{\circ} \mathrm{C}$ were never recorded, yet temperatures above $37^{\circ} \mathrm{C}$ were not uncommon.

Ambient temperature fell at the same time as rectal temperature with a similar, though shallower, time course, showing a total fall of $4 \cdot 4^{\circ} \mathrm{C}$. The extent of the fall in rectal temperature in individuals did 


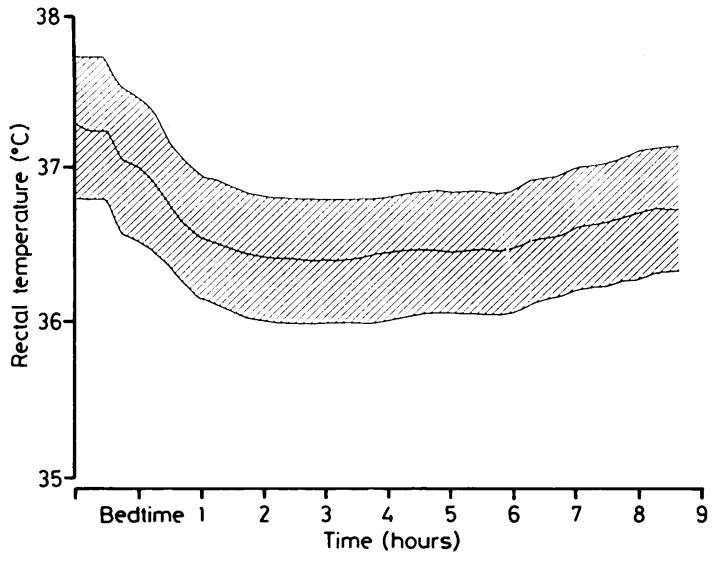

Fig 2 Normalised curve for rectal temperature changes over time after bedtime. A total of $90 \%$ of infants would be expected to fall within the shaded area on the assumption of a normal distribution.



Fig 3 Distribution of rectal temperatures across infants three hours after placing in their cot. Data do not depart significantly from a normal distribution but are slightly skewed to higher temperatures.

not, however, correlate either with the minimum ambient temperature $(r=-0 \cdot 2, n=64, p>0 \cdot 05)$ nor the fall in ambient temperature $(r=0.05, n=64$, $p>0 \cdot 1)$. Likewise, there was no correlation between rate of fall of rectal temperature and ambient temperature early in the night $(r=-0.06, n=64$, $\mathrm{p}>0 \cdot 1$ ).

The extent and rate of fall of rectal temperature also failed to correlate with the thermal insulation (tog value) of the infants' clothing and wrapping, so there is no evidence that the changes in rectal temperature are driven by the fall in ambient temperature.

\section{SKIN TEMPERATURE}

The temperature recorded by a thermistor on the skin of the abdomen did not reflect the pattern of rectal temperature. Fig 4 shows rectal and skin temperatures normalised to bedtime. Skin temperature was always lower than rectal, but varied much less over the night. The difference between skin and rectal temperature did, however, change appreciably.

The relation between skin and rectal temperature was examined by computing correlations between the two, both over successive measurements in the same individual, and over different individuals at the same time relative to bedtime. Over the whole night, fluctuation in abdominal skin and rectal temperature correlated significantly $(p<0.05)$ in only half of the children. There was a significant positive correlation in $19(28 \%)$, and a significant negative correlation in $15(22 \%)$. In the early part of the night rectal temperature was generally falling while skin temperature rose or remained stable, while late in the night both rose together. Which dominated determined the sign of the correlation if it existed. Restriction of the analysis to the final six hours of the recordings yielded significant positive correlations in $32(48 \%)$ of subjects.

If data are correlated across individuals at constant time, then there is at most times a highly significant (usually $\mathrm{p}<0 \cdot 001$ ) positive correlation with a slope close to 1 . During the half hour before and the hour after putting down, however, there is no significant



Fig 4 Rectal and skin temperatures of infants over the night. Data are normalised to bedtime. 
correlation. Skin temperature therefore only reliably predicts rectal temperature once the infant has settled in its cot, and furthermore during the first hour after putting down the difference between skin and rectal temperatures decreases at the same rate as rectal temperature declines.

\section{Discussion}

Although circadian rhythms of body temperature were described early in this century, ${ }^{1}$ there are few reports of continuous body temperature recordings in infants other than neonates, ${ }^{2-4}$ and we can find no recordings made under home conditions. The data reported here describe rectal and skin temperatures during sleep in a good size sample of 4 month old babies, and allow an adequate description of normality for this group.

There is a very consistent pattern across individuals. All but three of the 74 subjects showed a fall in rectal temperature, which is phase locked to placing in their cot and presumably therefore falling asleep. The three oddities will be discussed in a separate report. While the fall clearly occurs at night, it is not a circadian rhythm precisely locked to time of day as some infants were put down as early as 1930 and achieved a low rectal temperature by 2030, whereas others were not put down until 0100 , so their rectal temperature did not fall until 0230 . It is for this reason that we have defined normality relative to the time of putting down, rather than time of night, although we are not denying the existence of circadian rhythmicity, as reported by previous authors. ${ }^{1-4}$ It would be of great interest to discover what happens to rectal temperature during a sleeping period in day time.

Rectal temperature changes are only partly reflected by those of the skin. Up to two hours after putting to bed there is no relation between skin and rectal temperature either across or within individuals. This almost certainly reflects the impact of the greatly increased insulation provided by wrappings tending to inhibit skin cooling. Indeed, in some cases skin temperature rises appreciably. Later in the night skin and rectal temperature tend to change together, and each may be used to predict the other.

The phases of temperature change: rapid fall, stability, and slow rise are clearly part of a well orchestrated pattern, keeping temperature within narrowly defined limits irrespective of ambient temperature and insulation, suggesting that they are both internally driven and controlled. This should be taken into account when making clinical judgments based on spot temperature measurements taken during the night. Just before putting a baby down a temperature at the bottom end of the 'normal range' $\left(36 \cdot 2^{\circ} \mathrm{C}-37 \cdot 2^{\circ} \mathrm{C}\right)$ is very unlikely, whereas two hours later a reading at the top end of the range is equally unlikely.

This study has therefore allowed a full description of the normal pattern of temperatures in 4 month old babies. Such a pattern is not apparent at birth, so we must now investigate when it develops and what factors influence its first appearance.

This work was supported by the Foundation for the Study of Infant Deaths.

\section{References}

${ }^{1}$ Minors DS, Waterhouse JM. Development of circadian rhythms in infancy. In: Davies JA, Dobbing J, eds. Scientific foundations of paediatrics. London: Heinemann, 1982:980-97.

2 Hellbrügge T. The development of circadian rhythms in infancy. Cold Spring Harbor Symposium on Quantitative Biology 1960; 25:311-23.

${ }^{3}$ Hellbrügge T, Lange J, Rutenfranz J, Stehr K. Circadian periodicity of physiological functions in difference stages of infancy and childhood. Ann NY Acad Sci 1964;117:361-73.

${ }^{4}$ Mills JN. Development of circadian rhythms in infancy. Chronbiologia 1975;2:363-71.

5 Emery JL, Sunderland R. Febrile convulsions and eot death. Lancet 1981;ii:176-8.

6 Stanton AN. Overheating and cot death. Lancet 1984;ii:1199.

${ }^{7}$ Clulow E. Thermal insulation properties of fabrics. Textiles 1978;7:2.

${ }^{8}$ Wailoo MP, Petersen SA, Whittaker H, Goodenough P. The thermal environment in which 4 month old infants sleep at home. Arch Dis Child 1989;64:600-4.

Correspondence to Dr MP Wailoo, Department of Child Health, University of Leicester, PO Box 138, Leicester LE1 9HN.

Accepted 6 October 1988 